\title{
Are the Diagnosis and Treatment of Neuropsychiatric Comorbidity in Multiple Sclerosis Still in Their Infancy?
}

\author{
Jean-Marie Annoni, MD, ${ }^{1,2}$ Myriam Schluep, MD² and Pasquale Calabrese, MD, PhD ${ }^{3}$
}

1. Department of Neurology, and Medical School, Geneva University Hospitals; 2. Centre Hospitalier Universitaire Vaudois, Lausanne;

\author{
3. Department of Neurology, Bochum University Hospital
}

DOI: $10.17925 /$ USN.2008.04.01.19

Four clinical dimensions can be impaired in multiple sclerosis (MS): neurological function, cognition, emotions, and fatigue. Neurological impairment, cognitive deficits, and fatigue are recognized to be part of the functional handicap and deterioration of quality of life in MS patients, while the effect of emotional impairment, which includes behavioral changes and decision-making difficulties, has been less precisely defined. In this context, neuropsychiatric comorbidity secondary to MS is becoming an important issue in MS medical care, and therapeutic efforts strongly depend on these dimensions. Moreover, the chronic aspect of the disease, individual socio-demographic situations, and the pre-morbid characteristics of each subject, as well as the brain impairment itself, influence neuropsychiatric symptoms in MS and interact with one another. Hence, it is not a surprise that subjective quality of life in MS is dependent on all of these dimensions: for example, suicidal intent of MS patients is related not only to disease-induced mood disorders such as depression, but also to the lifestyle modifications induced by MS such as alcohol abuse and individual habits such as living alone. In addition, other medical parameters such as the MS therapy itself and biographical factors such as coping abilities may influence the ability to cope with and react to such neuropsychiatric symptoms.

In this article we will present some features of these different dimensions and propose diagnostic and therapeutic cures, the latter (both pharmacological and non-pharmacological) being promising but still in their infancy. However, the most effective treatment of neuropsychiatric symptoms at the moment is early causal MS therapy.

Fatigue, cognitive deficits, mood disorders, and affective changes are now recognized as frequent in early MS. It is crucial to differentiate the prevalence of subtle neuropsychiatric alterations in prospective cohort studies from clinically significant impairment, which must be diagnosed in clinical practice. However, prospective studies have shed light on the frequency and potentially devastating effect of such comorbidities, and have thus provided a rationale for efficient screening in everyday practice. We will focus separately on the different groups of symptoms in order to outline an operational approach to each of them.

Fatigue is a multidimensional, motor-perceptive, emotional, and cognitive experience, and in this sense it is highly representative of the neuropsychiatric symptoms found in MS. It can be independent of depression, but correlates highly with mood disorders, decreased levels of action control, and motivational disturbances. ' Objective fatigue-related factors (i.e. "observable and measurable decrement in performance occurring during the repetition of a physical or mental task") can be dissociated from subjective fatigue, which is characterized by "a feeling of early exhaustion, weariness, and aversion to efforts" similar to (although different from) neurasthenia, as defined by the 10th International Classification of Diseases (ICD-10). Moreover, in many ways fatigue in MS is qualitatively different from fatigue as experienced by stroke patients. It is characteristically relieved by rest and has a strong psychological impact. ${ }^{2}$

It is not surprising, given the diversity of manifestations of this symptom in MS, that evidence-based therapy for fatigue has been very disappointing, and most clinicians agree that the most successful approach is to adapt the activities of patients. However, there seem to be some pharmacological and non-pharmacological ways to alleviate fatigue symptoms. For example, clinical experience and systematic findings from some small series have suggested that a variety of symptomatic approaches-such as dopaminergic wake-promoting agents (e.g. methylphenidate or modafinil)—but also physical training, yoga, ${ }^{3}$ or acting on secondary causes - such as reduction of neurogenic pain, management of sleep difficulties, and treatment of depression-may have some beneficial effects in the treatment of fatigue. The challenge before editing guidelines to reflect this is a precise characterization of the type of fatigue for each MS patient.

Cognitive deficits have been estimated to occur in up to $70 \%$ of patients during the course of MS. They may also be the primary cause of secondary behavioral and emotional alterations. One example is the influence of impaired working memory on the ability to cope with stressful, complex situations. Cognitive dysfunctions may encompass a variety of functions, including long-term and working memory, interhemispheric transfer (which may lead to alexithymia), problem-solving, executive functions, information processing speed, and attention.

While the clinical profile of MS-related cognitive disturbances is characterized by a subcortical type of impairment, affecting mainly memory and executive functions, the range of neuropsychological deficits

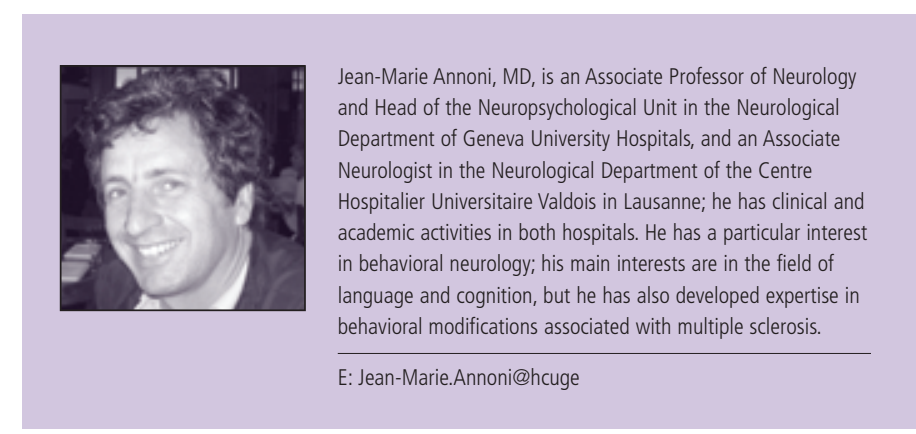


encountered in MS have been termed 'multiple disconnection syndrome, '4 indicating that the cognitive dysfunction seems to be due to manifold interruptions of different interacting fiber systems, leading to mulitiple deficits. Such deficits have also been evidenced in early MS, although the reported prevalences were variable, ranging from $25 \%$ to more than $50 \% .^{5}$ Incipient cognitive deficits have also been assessed longitudinally, suggesting that such deficits might be used as predictive parameters of MS evolution and severity.

This impairment can be caused either by a general slowing of information processing or by specific alterations of memory and executive functions. In the everyday life of MS patients, impairment of memory, attention, and concentration faculties, coupled with fatigue, directly affect their personal organization, as well as their vocational functioning and normal family role. Patients who are cognitively impaired are less likely to be working, are engaged in fewer social and vocational activities, report more sexual dysfunction, experience greater difficulty in performing household tasks, and exhibit more psychopathology than cognitively intact patients. ${ }^{6}$

Functional decline, stressful life events, and coping responses may have longlasting psychological implications in multiple sclerosis, just as they do in other chronic illnesses characterized by unpredictable and often poorly controlled functional decline.

Pharmacotherapy of cognitive disorders in MS has included a controlled trial of donepezil including 69 subjects; this trial found that donepezil improved verbal learning and memory compared with placebo during neuropsychological testing. ${ }^{7}$ However, such approaches are in their infancy.

Cognitive rehabilitation in MS is still underdeveloped. Much of our knowledge of cognitive rehabilitation strategies is derived from classic studies with brain-damaged subjects who for the most part had experienced acute brain injury ${ }^{8}$ or stroke. ${ }^{9}$ Thus, these rehabilitation approaches deal with the management of diseases that are of an acute onset and stable course. In this case, the predictions about the dynamics of recovery and the determinants of rehabilitation of both the cognitive and physical functions may be of greater reliability, since the set of dysfunctions under treatment have a more constant relationship. In the case of MS this constancy is not given, since the occurrence of new lesions, as well as the reactivation of temporary 'silent' lesions and, ultimately, the topical expansion of a given lesion, creates a highly dynamic pattern of brain involvement, at least in the active phase of the disease. This is also reflected at the behavioral level, where a variability and fluctuation of symptoms is common. Thus, while in acute brain injury the sequelae can be restricted to particular physical and cognitive domains, which in turn can produce only time-limited consequences, in a chronic disabling disease such as MS the treatment strategy has to take into consideration the aspect of chronicity.
Nevertheless, there are some promising results, such as self-generating learning approaches $^{10}$ and training protocols using personal digital assistants. However, according to a recent meta-analysis ${ }^{11}$ there are only 16 systematic studies of cognitive rehabilitation for persons with MS, only two of which provide practical guidelines. The main problem is that such studies are difficult to respect in terms of evidence-based principles, lengthy, less fashionable than other neuroscience research, and more difficult to publish in high-impact journals. Sponsoring of non-pharmacological interventional studies needs to be encouraged by health policy.

Functional decline, stressful life events, and coping responses may have longlasting psychological implications in MS, just as they do in other chronic illnesses characterized by unpredictable and often poorly controlled functional decline. However, organic brain dysfunction seems to play an important role in MS affective modifications.12 Although classically described, depression always seems underdiagnosed by neurologists. According to Mohr, $26 \%$ of 260 MS outpatients treated by 35 neurologists met Diagnostic and Statistical Manual of Mental Disorders, Fourth Edition (DSMIV) criteria for major depression. Interestingly, of these patients $66 \%$ received no antidepressant medication. This suggests that, despite the presence of reliable screening tools to detect depression in MS patients, screening is not yet widely enough adopted, ${ }^{13}$ a situation that should be changed since inability to cope with stressful events can worsen MS. One possible explanation for this situation is a sort of fatality when confronted by depressive symptoms. Thus, it must be borne in mind that antidepressants reduce the severity of depressive symptoms in MS patients and that controlled trials have demonstrated the effectiveness of cognitive behavioral therapy (CBT) in such patients.

Generalized anxiety disorders seem to be 20 times more common in MS patients than in control subjects, and there is double the frequency of alcohol abuse in the former than in the latter. ${ }^{12}$ If mood disturbance is the most commonly reported affective disorder in MS, behavioral manifestations have also been described as a variety of personality changes, although it is not possible to identify an 'MS personality.' According to Diaz-Olavarrieta, agitation (40\% occurrence), irritability (35\%), apathy (20\%), and disinhibition (13\%) are more frequent in MS patients. ${ }^{14}$ Behavioral manifestations, classically recognized as a variety of mood-related symptoms, include cheerfulness, optimism, denial of disability, and pathological laughing. These behavioral alterations are thought to represent corollary causes of central nervous system (CNS) pathology secondary to MS, and are rather late symptoms of the disease-although they were once considered to be the hallmark of MS. In particular, euphoric mood aberrations seem to be substrate-dependent disturbances, and tend to evolve in parallel with MS duration and severity. Other behavioral changes such as agitation, irritability, apathy, and disinhibition are more frequent and also seem to play an important role in MS perceived handicap. Interestingly, such behavioral modifications not only seem to be due to the neurological characteristics of MS, but also share similarities with chronic non-neurological diseases, suggesting that the chronicity itself plays an important role. ${ }^{15}$

Finally, MS patients, especially those individuals with a relapsingremitting disease course (RRMS), show impaired decision-making abilities. Their decreased learning proficiency in the lowa Gambling Task suggests that poor decision-making is a consequence of impaired learning across the trials. The key point of these observations is that impaired emotional experience, more than cognitive dysfunction, is associated with impaired lowa Gambling 
Task performances in RRMS patients. ${ }^{16}$ Moreover, some studies by Nagy et al. suggest that patients with RRMS show a similar performance to that observed in subjects with ventromedial prefrontal damage, namely that their decisions are guided by recent outcomes irrespective of gain or loss. ${ }^{17}$ Given the practical consequences of impaired decision-making, the management of such problems may include behavioral as well as interpersonal approaches.

Fortunately, much emphasis has been given recently to making the diagnosis of MS as early as possible ${ }^{18}$ in order to allow early immunomodulatory treatment induction, with the aim of avoiding the risk of further disease progression. However, MS remains a chronic disorder with a mostly unpredictable course and outcome. In a chronic disorder such as MS, with gradual onset, uncertain course, and periods of deterioration and remission, patients are at risk for developing anxiety and depression that are futureoriented, that is with fear of the unknown. Such anxious reactions may even worsen MS. ${ }^{19}$ Adaptation to this type of progressive disability includes several intervening factors, such as cognitive impairment, timing of the impairment, pre-morbid coping mechanisms and skills, severity of the functional handicaps, availability of a supportive familial network, and social resources. In particular, the development of specific adjustment strategies by individuals facing a stressful situation with problem-oriented coping seems to lead to better shortterm adjustment and a lower degree of disability. ${ }^{20,21}$

Therefore, disease-modifying therapies, including the immunomodulators broadly used in RRMS, are mandatory to prevent neuropsychiatric symptoms in MS. The intriguing observation is that some of these drugs are suspected to have (minor) influences on mood. Only interferon beta, in comparison with glatiramer acetate and natalizumab, has so far been suspected to induce mood alterations when evaluated in the early double-blind, placebo-controlled trials. However, more recent data support the absence of emotional worsening in MS patients treated with interferon beta for a long period of time, and emphasise the role of increased disability and the presence of baseline depressive symptoms predicting the presence of depressive symptoms at follow-up. ${ }^{22}$

\section{Acknowledgments}

Supported by grants from the Swiss Society for Multiple Sclerosis and the Swiss National Science Foundation (3200BO-104051 and 325100-118362).

\section{National Multiple Sclerosis Society Supporting Research in 2008}

The National Multiple Sclerosis Society (NMSS) continues to support and stimulate world-class research into ways to prevent, better treat, and cure MS. In spring 2008, the NMSS announced the commitment of over $\$ 24$ million to support 61 innovative MS research projects. Selected on scientific merit and relevance to the fight against MS, the new projects are part of a comprehensive research program slated to invest nearly $\$ 50$ million in 2008, including funding over 440 new and ongoing MS investigations in the US and abroad. By the end of fiscal year 2008, the NMSS will have invested $\$ 610$ million since its foundation to support basic and clinical research in MS.

Key to guiding the Society's funding decisions are three key pillars: speed, collaboration, and possibilities. Some key funding mechanisms and opportunities include:

- grants-multiyear investigations by university-based scientists for basic and clinical research;

- collaborative MS research center awards-five-year grants to interdisciplinary teams to stimulate cross-pollination of ideas and attract new minds to the field;

- high-risk/high-potential pilot grants-one-year awards to test innovative, cutting-edge ideas;

- industry partnerships-milestone-driven drug development funding for private companies;

- fellowships - to attract and train promising young investigators and doctors to focus on MS;

- rehabilitation research fellowships - to address the unmet need for specialists trained to conduct quality rehabilitation research; and

- healthcare delivery and policy contracts - to support key questions in healthcare delivery and policy research to inform advocacy efforts and enhance quality of life for people with MS.

For further information regarding the activities of the NMSS, please visit www.nationalmssociety.org

Source: National Multiple Sclerosis Society.
1. Penner IK, Bechtel N, Raselli C, et al., Fatigue in multiple sclerosis: relation to depression, physical impairment, personality and action control, Mult Scler, 2007;13(9):1161-7.

2. Gramigna S, Schluep M, Staub F, et al., Dimensions multiples de la fatigue d'origine neurologique: différences entre l'accident vasculaire cérébral et la sclérose en plaques, Rev Neurol (Paris), 2007;163(3):341-8.

3. Oken BS, Kishiyama S, Zajdel D, et al., Randomized controlled tria of yoga and exercise in multiple sclerosis, Neurology, 2004;62(11): 2058-64.

4. Calabrese P, Penner IK, Cognitive dysfunctions in multiple sclerosis-a "multiple disconnection syndrome"?, J Neurol, 2007;254(Suppl. 2):II18-21.

5. Simioni $S$, Annoni JM, Bruggimann $L$, et al., Cognitive impairement is associated with mood disorders and fatigue at the early stage of multiple sclerosis, Swiss Med Wkly, 2007;137(35-36):496-501.

6. Rao SM, Leo GJ, Ellington L, et al., Cognitive dysfunction in multiple sclerosis. II. Impact on employment and social functioning, Neurology, 1991;41:692-6.

7. Christodoulou C, Melville P, Scherl WF, et al., Effects of donepezil on memory and cognition in multiple sclerosis, J Neurol Sci, 2006;245(1-2):127-36.
8. Prigatano GP, Fordyce DJ, Zeiner HK, et al., Neuropsychological rehabilitation after closed head injury in young adults, J Neurol Neurosurg Psychiatry, 1984;47(5):505-13.

9. Wade DT, Wood VA, Hewer RL, Recovery after stroke- the first 3 months, I Neurol Neurosurg Psychiatry, 1985;48(1):7-13.

10. Basso MR, Ghormley C, Lowery N, et al., Self-generated learning in people with multiple sclerosis: An extension of Chiaravalloti and DeLuca (2002), J Clin Exp Neuropsychol, 2007;12:1-7.

11. O'Brien AR, Chiaravalloti N, Goverover Y, Deluca J, Evidence-based cognitive rehabilitation for persons with multiple sclerosis: a review of the literature, Arch Phys Med Rehabil, 2008;89(4): 761-9.

12. Chwastiak LA, Ehde DM, Psychiatric issues in multiple sclerosis, Psychiatr Clin North Am, 2007;30(4):803-17.

13. Mohr DC, Hart SL, Julian L, Tasch ES, Screening for depression among patients with multiple sclerosis: two questions may be enough, Mult Scler, 2007;13(2):215-19.

14. Diaz-Olavarrieta C, Cummings JL, Velazquez J, Garcia de al Cadena C, Neuropsychiatric manifestations of multiple sclerosis, I Neuropsychiatry Clin Neurosci, 1999;11:51-7.

15. Souza Lima F, Simioni S, Bruggimann L, et al., Perceived behavioral changes in early multiple sclerosis, Behav Neurol,
2007:18(2):81-90

16. Kleeberg J, Bruggimann L, Annoni JM, et al., Altered decisionmaking capacity in multiple sclerosis: a sign of impaired emotional reactivity? Ann Neurol, 2004:56:787-95.

17. Nagy H, Bencsik K, Rajda C, et al., The effects of reward and punishment contingencies on decision making in multiple sclerosis, I Int Neuropsychol Soc, 2006;12:559-65.

18. Polman CH, Reingold SC, Edan G, et al., Diagnostic criteria for multiple sclerosis: 2005 revisions to the "McDonald Criteria", Ann Neurol, 2005;58(6):840-46.

19. Mohr DC, Stress and multiple sclerosis, J Neurol, 2007;254 (Suppl. 2):॥65-8.

20. Pakenham Kl, Adjustment to multiple sclerosis: application of a stress and coping model, Health Psychol, 1999;18(4):383-92.

21. Pakenham KI, Making sense of illness or disability: the nature of sense making in multiple sclerosis (MS), J Health Psychol, 2008;13(1):93-105.

22. Porcel J, Sanchez-Betancourt A, Arévalo MJ, et al., Long-term emotional state of multiple sclerosis patients treated with interferon beta, Multiple Sclerosis, 2006;12:802-7. 